\title{
Design considerations for ease of access and maneuverability of OCT imaging platforms in the oral cavity
}

George Dobre

George Dobre, "Design considerations for ease of access and maneuverability of OCT imaging platforms in the oral cavity," Proc. SPIE 10831, Seventh International Conference on Lasers in Medicine, 108310F (10 August 2018); doi: 10.1117/12.2282646

Event: Seventh International Conference on Lasers in Medicine, 2017, Timisoara, Romania 


\title{
Design Considerations for ease of access and maneuverability of OCT Imaging Platforms in the Oral Cavity
}

\author{
George Dobre \\ Applied Optics Group, School of Physical Sciences, University of Kent, Canterbury, CT2 7NH, UK
}

\begin{abstract}
With the advent of instruments incorporating elements of confocal microscopy and Optical Coherence Tomography (OCT), optical technologies are steadily gaining usage in a variety of biomedical applications. They offer tissue visualization both at the superficial level and in depth, they operate using non-ionizing radiation at a relatively low cost, and the procedures are easily tolerated by patients. One area of growth for optical imaging is in the oral cavity, which has excellent optical accessibility both for dental tissue and oral mucosa. The need to image features accurately (both as part of oral mapping and as part of investigating the underlying tooth structure) requires producing 3-D volumes with sufficiently good lateral and vertical resolution to allow identification of features of interest.
\end{abstract}

Although a few types of intraoral optical scanners are already successfully commercialized for surface mapping, the examination of optical design considerations that are particular to scanned beam imaging in the oral cavity does highlight particular challenges of interest to both clinicians and optical engineers engaged in designing the next generation of optical instruments.

Keywords list: confocal microscopy, optical coherence tomography (OCT), intra-oral scanning, GRIN lens technology.

\section{INTRODUCTION}

Optical Coherence Tomography (OCT) is an interferometric technique capable of detecting light scattered from within tissue or from boundaries between different tissue layers. It can be used to obtain structural sub-surface morphology of regions situated up to a few mm inside tissue, as well as from the surface / air-tissue boundary (which can be of considerable interest in dental investigations [1]-[4]).

Optical instruments that can facilitate non-OCT type of confocal imaging of dental tissue in the oral cavity have been successfully commercialised, such as for example iTero (Align Technology), CEREC (Dentsply Sirona), Trios (3Shape) and several others [5],[6] but they rely on relatively high levels of confocality which is necessary to provide depth discrimination of the air-tissue boundary, with a resolution of around 10 microns.

OCT requires a probe beam and a reference beam. Lateral resolution is achieved by delivering the probe beam through a confocal channel of (typically) low to medium confocality (NA between 0.1 and 0.4 ), in order to maintain a reasonable depth of focus both at the target tissue and below the surface of the target tissue. Since OCT relies on interferometric rather than confocal depth sectioning, the emphasis is on lower magnification and better depth of focus for OCT imaging.

Apart from delivery through a confocal channel, the need to provide illumination and collection of backscattered light imposes its own constraints in OCT depth imaging. Exploring volumes within teeth and soft tissue in the oral cavity is achieved by scanning the optical beam laterally across the area of interest (which may be only as wide as an individual tooth, or, if mapping is desired, may extend across several teeth to a quadrant or an arch, which has been shown to be possible with 3D intraoral scanners). Regardless of the design, the optical conduit of such devices should have an exit aperture that is wide enough to accommodate the beam as it is being scanned ([7],[8]). For a beam diameter of 2-3 $\mathrm{mm}$ the exit aperture may need to accommodate the beam on axis, as well as off-axis at the extremes of the scan angle range,

Seventh International Conference on Lasers in Medicine, edited by Darinca Carmen Todea,

Adrian Gh. Podoleanu, Virgil-Florin Duma, Proc. of SPIE Vol. 10831, 108310F

(C) 2018 SPIE · CCC code: 1605-7422/18/\$18 · doi: 10.1117/12.2282646 
thereby increasing the exit aperture of the tip of the instrument which may be difficult to accommodate particularly for posterior teeth.

Information from up to several $\mathrm{mm}$ within tissue can be obtained in several wavelength windows, mostly in the near infrared wavelengths of $750 \mathrm{~nm}$ and longer which are suitable for deeper penetration in dental tissue and oral soft tissue, whose light attenuation properties due to water absorption should be taken into consideration. For in vivo imaging at faster speeds, Swept Source OCT (SS-OCT) configurations are often employed due to their higher sensitivity and generally faster scanning speeds.

In the context of imaging of oral cavity tissue, the design of the system delivering the probe beam to the desired volume of tissue (comprising tissue surface and a depth of 2-3 $\mathrm{mm}$ immediately below it), should be informed by the imaging resolution, lateral / depth of field size requirements and working distance as well as by the ease of access to parts of the oral cavity. These are often competing requirements, and typically the magnification of the confocal platform on which the OCT system is built is relatively low, in order to produce useful images of features in the $\mathrm{mm}$ range.

This paper examines the possibility of building a versatile optical column in the object arm of an OCT system, using common GRIN lens elements, with the purpose of allowing easy access to a large part of the oral cavity in a way that makes the investigative imaging procedure comfortable for the patient.

\section{DESIGN CONSIDERATIONS}

The key design consideration for the confocal microscopy platform employed to deliver the investigation beam to the tissue volume for imaging is the need to form the OCT image by scanning the beam laterally, usually at right angles to the direction of propagation of light. This is commonly accomplished by pivoting the beam around the axis of a galvanometer scanner situated at the entrance aperture of a telescope system, with the tissue located axially at the conjugate point of the telescope's exit aperture as described in the diagram in Fig.1. The length of the telescope system constitutes the bulk of the distance between scanner and tissue. Ideally the number of elements in the telescope should be kept to a minimum in order to avoid a loss of intensity due to stray reflections.

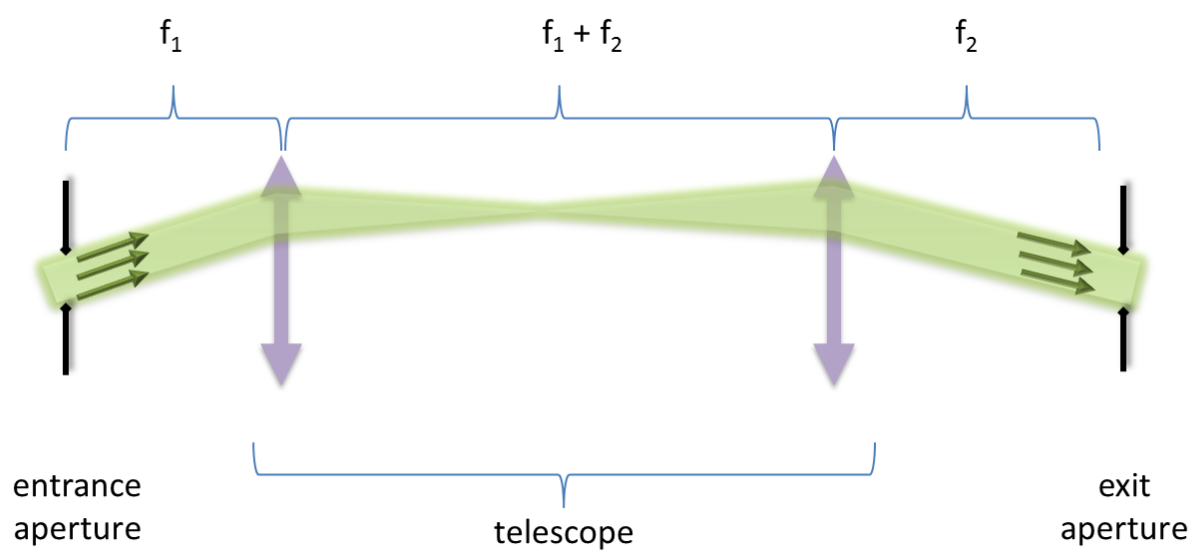

Figure 1. Scanning setup used to facilitate the lateral exploration of a the surface of interest. The pivot axis of the scanner is placed at the entrance aperture of the telescope consisting of refractive elements of focal lengths $f_{1}$ and $f_{2}$. The image plane is conjugate with the exit aperture of the telescope.

One specific set of design parameters to oral cavity imaging can be derived from the need to angle the imaging instrument around teeth in order to image either dental tissue (e.g. molars from the side) or oral soft tissue. Although insertion of wider tube is possible in the oral cavity, in order to avoid patient discomfort, the exit aperture at the distal 
end of the telescope should not be larger than a few $\mathrm{mm}$, in order to allow a small periscope-like arrangement to be fitted around the tooth profile.

For these reasons, the type of design proposed in this paper employs a telescope consisting of commonly available GRIN lenses and other appropriate small aperture optics not exceeding OD values of $3 \mathrm{~mm}$ (Fig. 2). GRIN lenses have been used in OCT imaging before [9]

To satisfy these requirements, the design represented in Fig. 2 is proposed. Modelled in Zemax OpticStudio ${ }^{\mathrm{TM}}$, it consists of an adjustable collimator arrangement composed of three singlet lenses: L1 (Edmund Optics 45-375), L2 (Edmund Optics 67-591) and L3 (Edmund Optics 45-273). These lenses have a $2.7 \mathrm{~mm}$ clear aperture and their judicious relative positioning allows a beam of light, consisting of wavelength components in the range 1030-1060 nm, and emerging from a single mode fibre, to be focused approximately $11 \mathrm{~mm}$ away from the exit aperture of L3. This is a sufficiently large clearance to allow for the positioning of at least one scanner mirror (as shown in the diagram in Fig.2) at a distance of $5.5 \mathrm{~mm}$ from L3. The clear aperture of the scanning mirror modelled in this study is $2.7 \mathrm{~mm}$. The design however allows for two suitably small scanning mirrors to be accommodated, thereby making it feasible for 3-D volumes of data to be obtained from the tissue of interest.

The scanned beam subsequently enters a sequence of four identical GRIN lenses (Thorlabs GRIN 2910, $1.8 \mathrm{~mm}$ diameter), whose purpose is twofold: a) to amplify the scanning angle in order to generate a suitable field size at the image plane and $b$ ) to ensure that the final lens can refocus the beam in a way that results in a quasi-uniform spot size at the image plane, across the desired lateral scanning range.

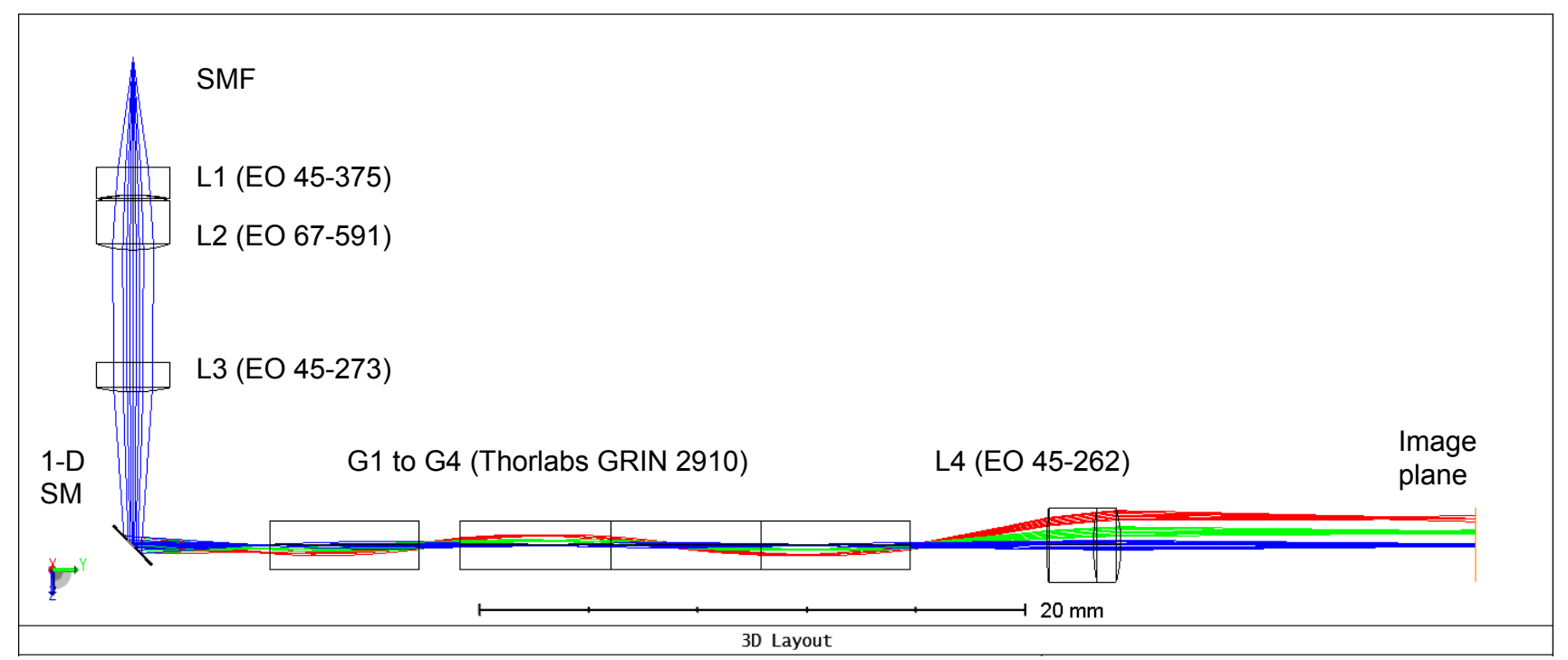

Figure 2. Layout of the proposed $3 \mathrm{~mm}$ diameter system capable of producing a lateral scan of $2.75 \mathrm{~mm}$ with 40 micron lateral resolution. SMF: single mode fibre; L1-L3: singlet lenses; 1-D SM: one dimensional scanning mirror; G1-G4: GRIN lenses, $1.8 \mathrm{~mm}$ diameter; L4: achromatic lens. The pivot axis of 1-D SM is placed at the entrance aperture of the telescope consisting of G1-G4. The image plane is conjugate with a point situated inside G4.

To enhance the simplicity of the design and reduce the number of stray reflections, the first of the four GRIN elements is set apart while the other three are grouped together with zero separation. At the exit aperture of the GRIN lens system, the beams emerge with amplified scan angles and with a minimal amount of divergence (more pronounced at the 0 scan angle). The final lens in the system is a $2.7 \mathrm{~mm}$ clear aperture achromat (Edmund Optics 45-262) which serves as the objective lens of the confocal platform and focuses the beam on the tissue.

The maximum scanner angle (which in turn determines the lateral field size) is limited by the aperture of lens L4. The choice of a relatively small $2.7 \mathrm{~mm}$ clear aperture is imposed by the need for easy access to the surface of oral tissue, which requires a compromise to be made on the maximum achievable extent of the lateral field size. The interval between L4 and the image plane is where any periscope mirror or prism would need to be placed, therefore L4's nominal focal length needs to be sufficiently large to allow this. A representation of the possible placement of this periscopic end mirror is shown in Fig.3. 


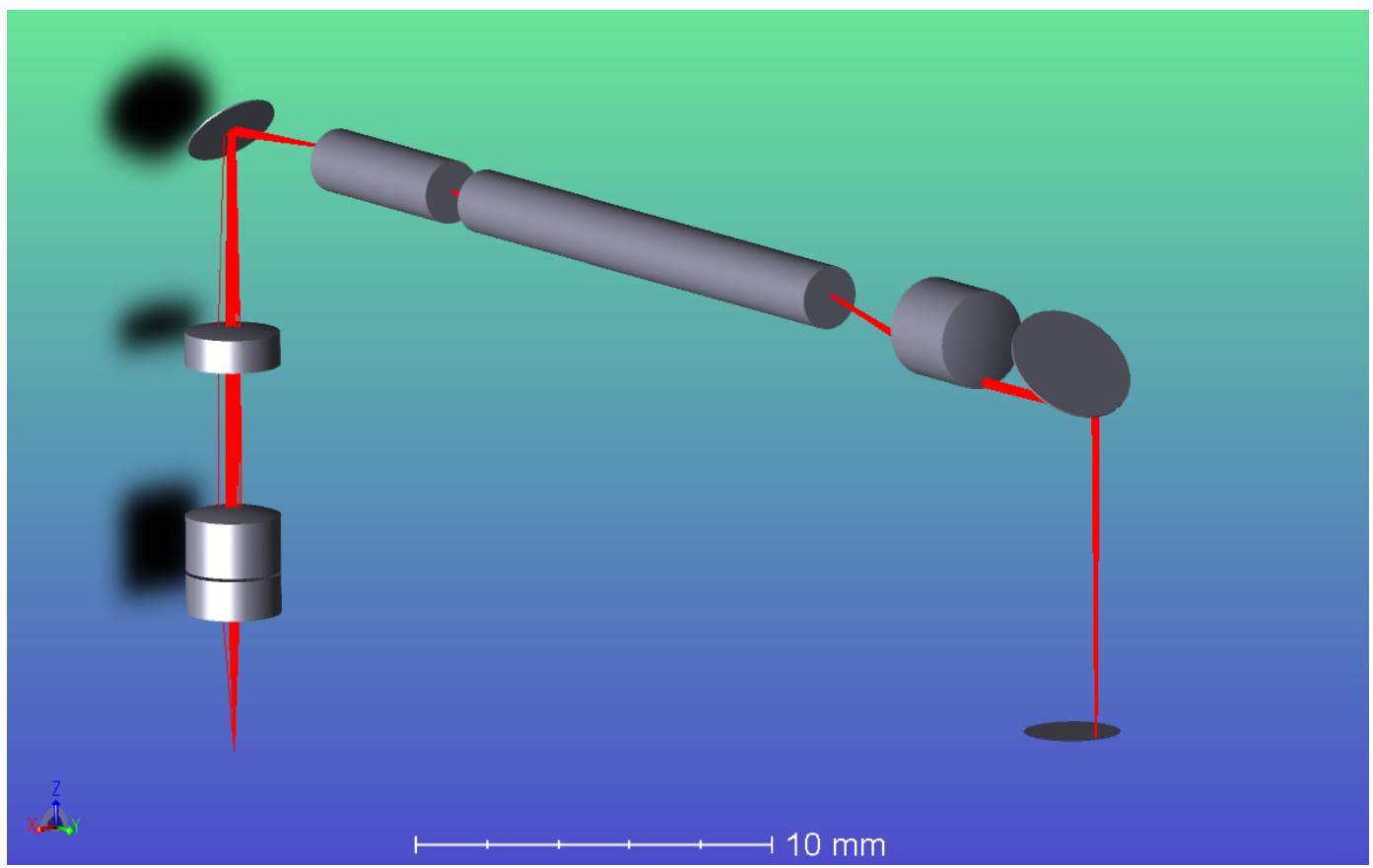

Figure 3. Shaded model representation of the scanning optical system proposed, to which one or two periscopic end mirrors can be added to aid access around dental tissue. The direction of propagation of the light beam shown corresponds to the maximum lateral extent of a half-scan ( $1.35 \mathrm{~mm}$ half field, $2.7 \mathrm{~mm}$ full field)

\subsection{Spot size analysis}

\section{ANALYSIS}

The maximum achievable lateral imaging resolution of the proposed imaging system is determined by the largest of RMS spot radius or the Airy disk radius. Three configurations have been modelled in Zemax OpticStudio ${ }^{\mathrm{TM}}$ and considered for analysis (1, 2 and 3), corresponding to different scan angles spanning half the field (from $0 \%$ to $100 \%$; while the lower half, from $-100 \%$ to $0 \%$, is assumed to result in an identical performance due to symmetry considerations).

The table below illustrates the relationship between the angle of the scanner mirror, the angle of the beam emerging from the final GRIN element, and the relative field position in the image plane (expressed as percentage of the image field, with the maximum being set to 1 ).

\begin{tabular}{|c|c|c|c|c|}
\hline Configuration & $\begin{array}{c}\text { Chief ray } \\
\text { orientation relative } \\
\text { to optical axis }\left({ }^{\circ}\right) \\
\text { after reflection on } \\
\text { scanner mirror } \\
\end{array}$ & $\begin{array}{c}\text { Chief ray } \\
\text { orientation relative } \\
\text { to optical axis }\left({ }^{\circ}\right) \\
\text { on exiting final } \\
\text { GRIN surface } \\
\end{array}$ & $\begin{array}{l}\text { Relative field } \\
\text { position (as } \\
\text { a percentage } \\
\text { of maximum) }\end{array}$ & Diagram \\
\hline 1 & 0 & 0 & 0 & $=1$ \\
\hline & & & & 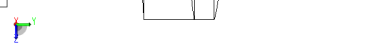 \\
\hline 2 & 0.9 & 5.16 & $52.4 \%$ & 1 \\
\hline 3 & 1.8 & 10.49 & $100 \%$ & \\
\hline
\end{tabular}


Spot size analysis at the image plane was carried out in for each of the three configurations (Fig. 4). It reveals that while there is a small variation in the Airy radius across the full extent of the lateral field between $38.8 \mu \mathrm{m}$ (Config. 1) and $36.4 \mu \mathrm{m}$ (Config. 3), the RMS spot size does not change with wavelength in any given configuration by more than about $10 \%$ between the limits of the OCT imaging spectrum, i.e. $1030 \mathrm{~nm}$ and $1070 \mathrm{~nm}$.
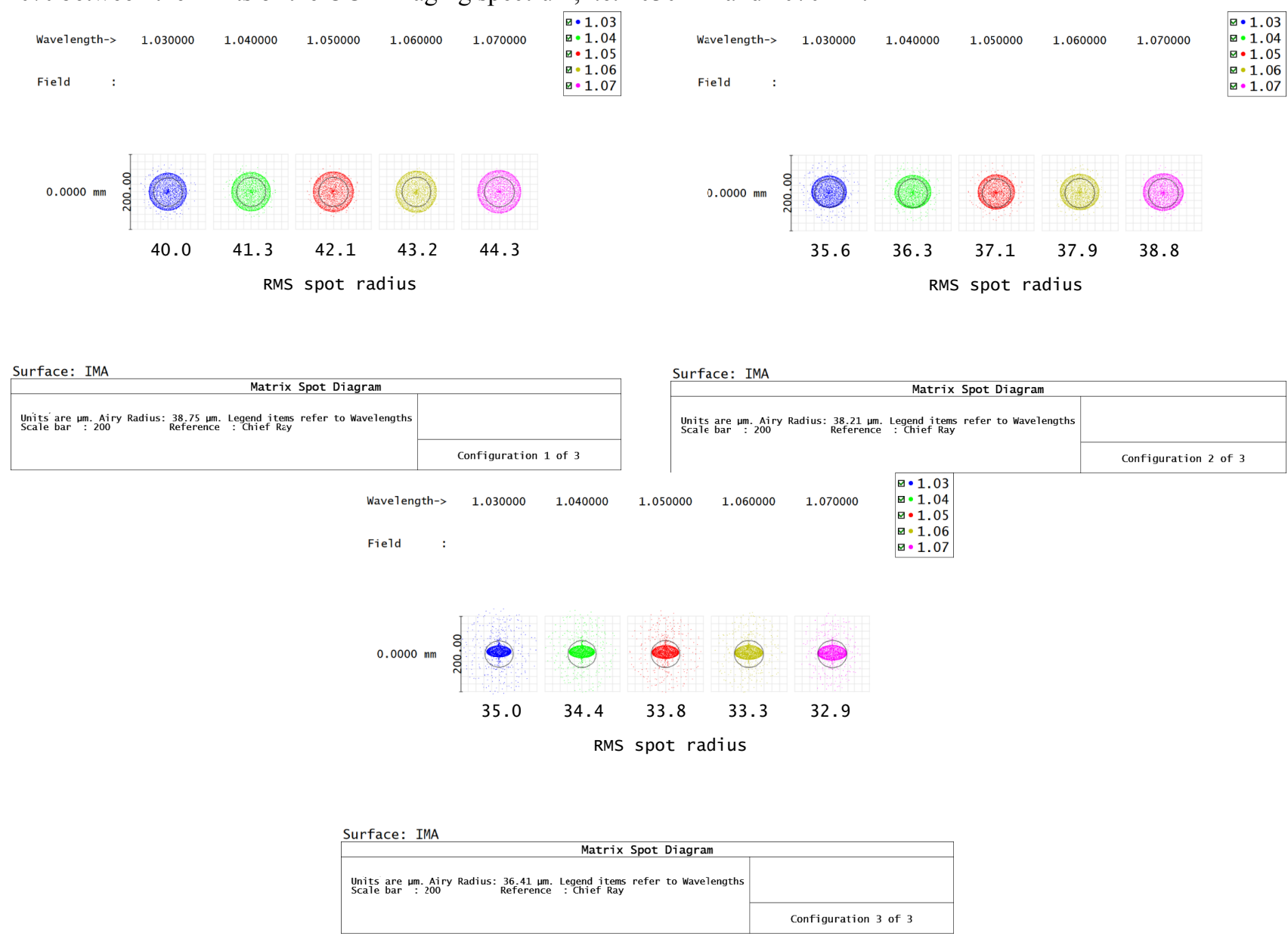

Figure 4. Spot size analysis for each of Configurations 1, 2 and 3. The RMS spot radius is shown for each configuration / scan angles, at five distinct wavelengths spanning the OCT imaging spectrum. The Airy disk is shown as a dark outline and a good match is notable between the RMS spot size and the Airy disk size in each case.

The mismatch, albeit small, between the Airy radius and the RMS radius changes (and inverts) as the scan angle progressively increases. In the centre of the field, at $0^{\circ}$ (Config. 1) spots are aberration limited with the greatest mismatch showing for $1070 \mathrm{~nm}$ where the RMS spot size is $44.3 \mu \mathrm{m}$ ( $\mathrm{cf} 38.74 \mu \mathrm{m}$ Airy radius). At the edge of the field, at a scan angle of $1.8^{\circ}$ (Config. 3) the Airy radius is larger than all RMS spot radii so the imaging regime is diffraction limited. This shows that the design is performing close to or at almost exactly the limits imposed by the physical constraints, i.e. aperture and working distance and that there is no advantage in optimizing for aberrations any further.

\subsection{Variation of spot size in the axial direction}

Given that the system is intended to produce depth images, one important parameter is the depth of focus, which is a measure of the range of depths over which the focusing is still sufficiently sharp, without refocusing adjustments needing to be carried out. 
The depth of focus is in an inverse relationship with the lateral resolution: worse lateral resolution, i.e. a larger minimum resolvable feature size, results in better depth of focus. Low numerical aperture focusing optics are generally used to achieve this. The analysis of spot variation in the axial direction was carried out with the aid of the "through focus spot size" diagrams shown in Fig. 5. In these diagrams, a virtual "defocus" is achieved by stepping the image plane in increments of $500 \mu \mathrm{m}$. The spot size at the resulting axial position is shown for each of the three configurations in Fig. 5.

$$
\varpi \cdot 1
$$
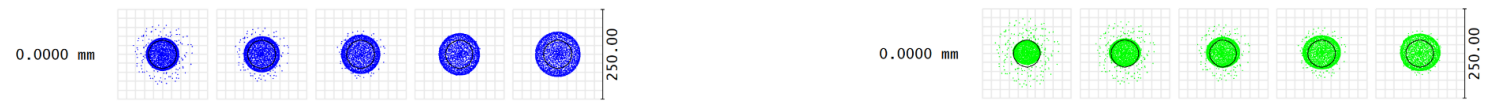

$-1000 \quad-500<-$ Defocus in um $-500 \quad 1000$
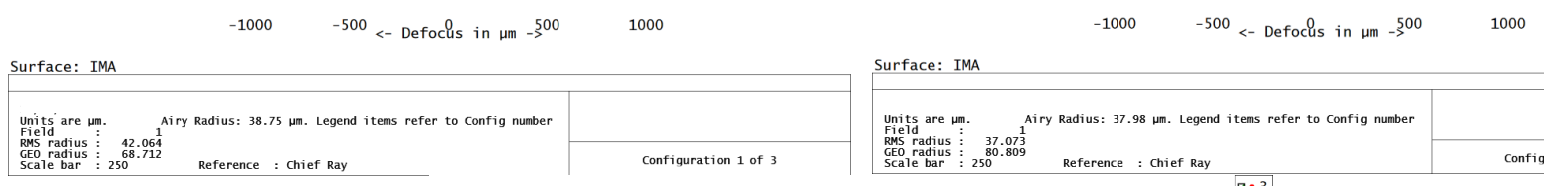

Surface: IMA
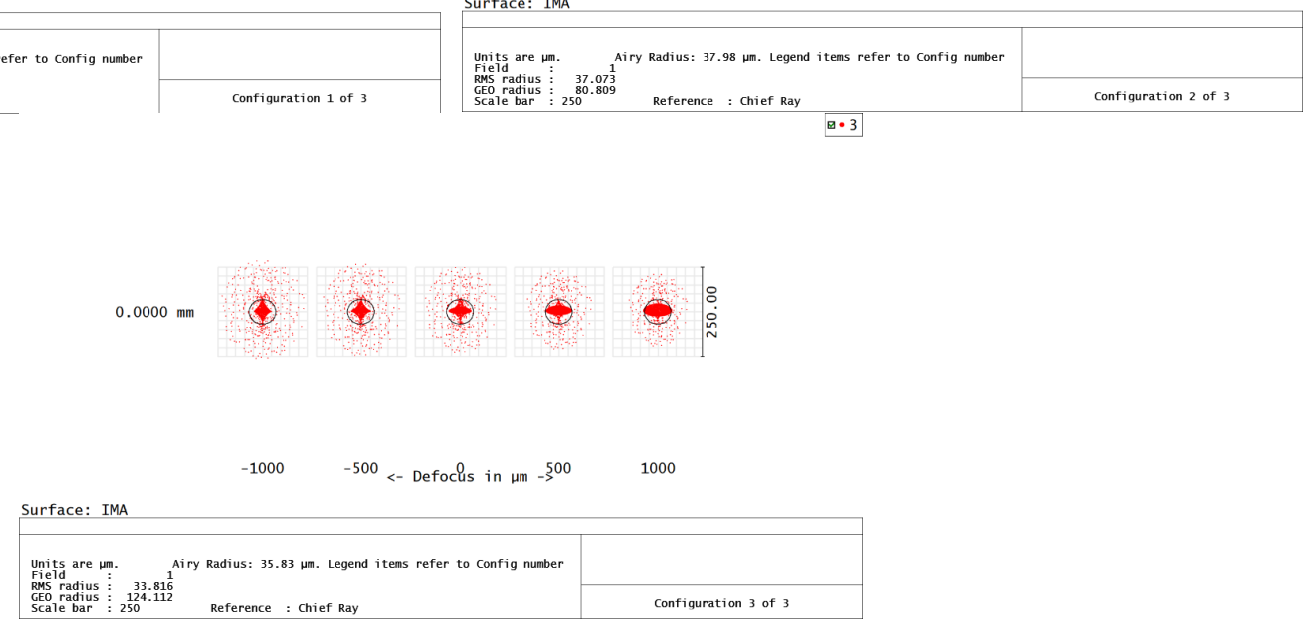

Figure 5. Through focus spot size analysis for each of Configurations 1, 2 and 3. Defocus is modelled over a total distance of $2 \mathrm{~mm}$ ( $1 \mathrm{~mm}$ away in either direction from the axial position of the image plane)

The analysis reveals that depth of focus is substantially similar regardless of configuration (i.e. scan angle), and the axial depth range over which the spot size does not exceed $50 \mu \mathrm{m}$ radius extends considerably beyond the modelled $2 \mathrm{~mm}$ (which would be sufficient for OCT imaging), and in fact the spot radius continues to be diffraction limited in Configurations 2 and 3 over that distance.

\subsection{Telecentricity and field curvature}

The imaging of highly reflective dental tissue presents the challenge that the backscattered light may be non-uniform in intensity, with significant discrepancies between the centre and the edge of the field. For this reason it is desirable to ensure consistent illumination angles as close as possible to normal incidence on the tooth surface, and there is an advantage in producing 1-D B-scans in the sagittal plane where the tooth curvature is less pronounced, rather than in the transverse plane.

To accomplish this, lens L4 should operate as much as possible like a telecentric $f$ theta lens (Fig. 6), so that the illumination of the image plane is consistently close to the normal orientation, and light scattered by tissue and returned to the originating fibre is of a similar intensity across the entire scan range.

Field curvature is another important parameter because it can result in defocus at the edges of the field. However, the relatively small angles of scan employed in this design keep field curvature values limited to less than $1 \mathrm{~mm}$ over the scan range, and given the $>2 \mathrm{~mm}$ depth of focus over which diffraction limited imaging is possible, the actual curvature results in a much less severe impact on the sharpness of OCT images. 

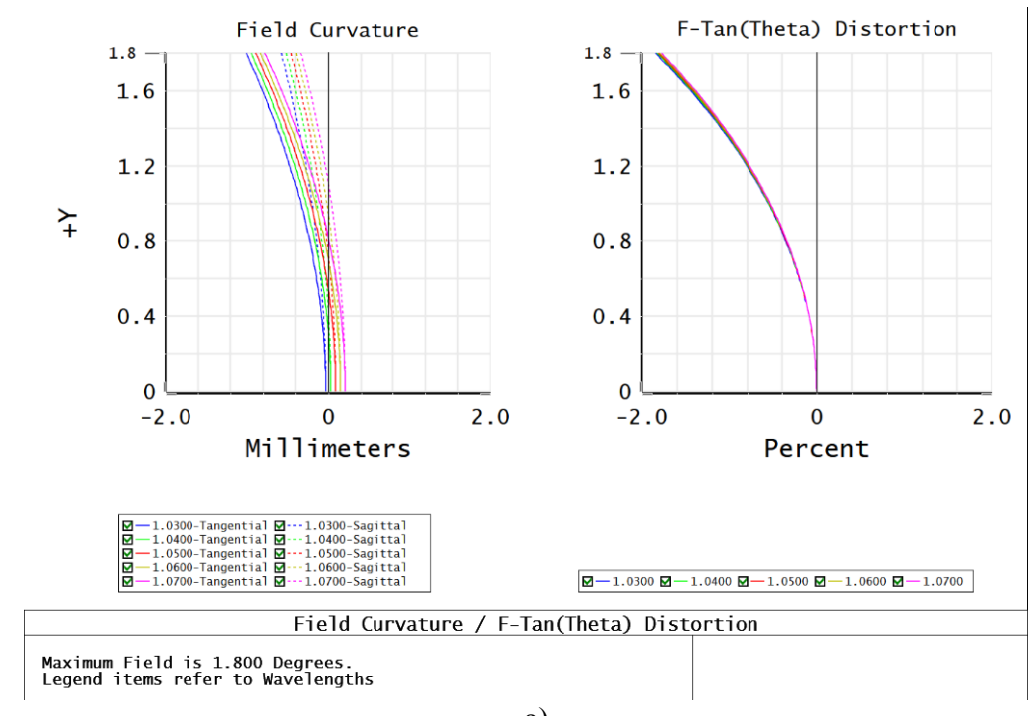

a)

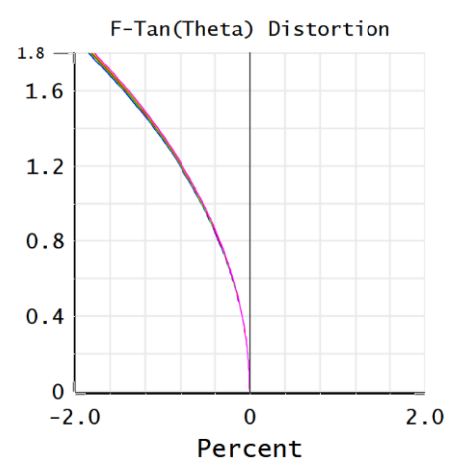

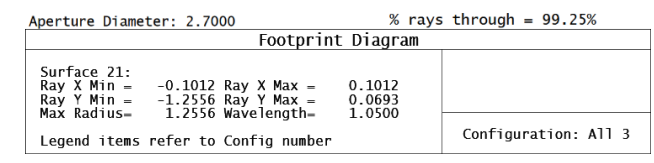

b)

Figure 6. a) Field curvature in the image plane. The maximum field curvature is just under $1 \mathrm{~mm}$ at the edge of the field (corresponding to a $+\mathrm{Y}$ value of 1.8); b) Footprint diagram of beams corresponding to each the three configurations striking the image plane. The edge of the field is situated at a distance of $1.135 \mathrm{~mm}$ from the centre of the field.

\section{CONCLUSIONS}

A very compact and easily manoeuvrable scanning system for OCT imaging in the oral cavity is proposed. The system has an optical column of $3 \mathrm{~mm}$ outside diameter, and is capable of lateral resolution of 35-40 $\mu \mathrm{m}$ (mostly diffraction limited) over a depth of $4 \mathrm{~mm}$. The electrically actuated scanner is located near the operator (proximal) end and the optical elements are all common stock items so this design may offer the possibility of lower complexity and cost in comparison to designs that use a moving mirror situated close to the distal end, close to the tissue of interest.

The system operates near the area in parameter space where diffraction and aberrations impose very similar limits to the achievable resolution. The choice of objective lens (L4) influences the size of the Airy disk, which determines the maximum achievable resolution in the diffraction limited regime of operation. If the outside diameter of the elements forming the optical column is set to $3 \mathrm{~mm}$, and assuming a working distance of at least $10 \mathrm{~mm}$ to allow for positioning of the final mirror in order to access hard-to-reach tissue surfaces, the Airy disk radius at the image plane cannot be significantly smaller than about $35 \mu \mathrm{m}$. In these circumstances, optical aberrations introduced by the GRIN elements are not a factor and hence performance improvements cannot be obtained by reducing the aberration-limited spot radius beyond this level.

The implication of this finding is that for imaging through the proposed system having an exit aperture value of $2.7 \mathrm{~mm}$ and a working distance of $10-12 \mathrm{~mm}$ (lens L4) there is no advantage in optimising for the aberrations unavoidably introduced by the four GRIN elements. The choice of GRIN optics to provide the telescopic arrangement is therefore justified - even though the combined aberrations are at a relatively high level.

This would be different if the diameter of the tube could be increased by a factor of 2 , in which case there would be an opportunity to reduce the RMS spot size to a smaller value through optimisation of the GRIN optics and/or of the final objective lens. Similarly there would be an advantage in optimising for aberrations if two separate scanners (X and Y) were to be employed. In this situation the scan angles in both directions should be matched to the maximum available rectangular area that can be circumscribed by the final lens; if a larger lateral field size is desired, there should be a corresponding increase in the exit aperture of (at least) lens L4 in order to accommodate this rectangular aspect. In order 
to mitigate against this, while retaining the advantage of a small aperture, a suitable design for the telescopic system dual axis scanner may be designed for particular tissue imaging in a non-telecentric way that allows a larger field, similar to the way in which some rod endoscopes operate.

\section{ACKNOWLEDGMENTS}

The author acknowledges the support of the Romanian National Authority for Scientific Research, CNDI-UEFISCDI project PN-III-P2-2.1-PED-2016-1937 (http://3om-group-optomechatronics.ro/).

\section{REFERENCES}

[1] Wang, X.J., Milner, T.E., de Boer, J.F., Zhang, Y., Pashley, D.H., Nelson, J.S., "Characterization of dentin and enamel by use of optical coherence tomography", Applied Optics 38(10), pp.2092-6 (1999)

[2] Amaechi, B.T., Higham, S.M., Podoleanu, A.Gh., Rogers, J.A., Jackson, D.A., "Use of optical coherence tomography for assessment of dental caries: quantitative procedure", Journal of Oral Rehabilitation, 28(12), pp.1092-3 (2001)

[3] Sinescu, C, Duma, V.-F., Canjau, S, Dobre, G, Demian, D, Cernat, R, Negrutiu M. L., Todea, C, Topala, F. I., Hutiu, Gh., Bradu, A., Podoleanu, A. Gh., "Dentistry investigations of teeth and dental prostheses using OCT", Proc. SPIE 9887, 98870B (2016)

[4] Yoshioka, T., Sakaue, H., Ishimura, H., Ebihara, A., Suda, H., Sumi, Y., "Detection of root surface fractures with swept-source optical coherence tomography (SS-OCT)" Photomedicine and Laser Surgery, 31(1) pp.23-7 (2013)

[5] Nedelcu, R.G., Persson, A.S., "Scanning accuracy and precision in 4 intraoral scanners: an in vitro comparison based on 3-dimensional analysis". Journal of Prosthetic Dentistry, 112, pp.1461-1471 (2014)

[6] Renne, W.G., Ludlow, M.E., Fryml, J., Schurch, Z., Mennito, A.S., Kessler, R., \& Lauer, A. "Evaluation of the accuracy of 7 digital scanners: An in vitro analysis based on 3-dimensional comparisons", Journal of Prosthetic Dentistry, 118 1, 36-42 (2017).

[7] Duma V.-F., Dobre, G., Demian, D., Cernat R., Sinescu C., Topala F. I., Negrutiu M. L., Hutiu Gh., Bradu A., Podoleanu A. Gh., "Handheld scanning probes for optical coherence tomography", Romanian Reports in Physics, 67(4), pp.1346-58 (2015).

[8] Duma V.-F., Dobre, G., Demian, D., Cernat R., Sinescu C., Topala F. I., Negrutiu M. L., Hutiu Gh., Bradu A., Rolland J. P., Podoleanu A. Gh., "Handheld probes and galvanometer scanning for optical coherence tomography", Proc. SPIE 9626, 96261J (2015).

[9] Xie, T., Guo, S., Chen, Z., Mukai, D., Brenner, M., "GRIN lens rod based probe for endoscopic spectral domain optical coherence tomography with fast dynamic focus tracking," Optics Express 14, 3238-3246 (2006) 